Review Article

\title{
Immunotherapy for Hepatocellular Carcinoma: Current Advances and Future Expectations
}

\author{
Yingjun Xie $\left(\mathbb{D},{ }^{1,2}\right.$ Yien Xiang, ${ }^{1}$ Jiyao Sheng $\left(\mathbb{D},{ }^{1}\right.$ Dan Zhang, ${ }^{1,2}$ Xiaoxiao Yao, ${ }^{1}$ \\ Yongsheng Yang $\mathbb{D}^{1,2}$ and Xuewen Zhang $\mathbb{D}^{1,2}$ \\ ${ }^{1}$ Department of Hepatobiliary and Pancreatic Surgery, Second Hospital of Jilin University, Changchun 130041, China \\ ${ }^{2}$ Jilin Engineering Laboratory for Translational Medicine of Hepatobiliary and Pancreatic Diseases, Changchun 130041, China \\ Correspondence should be addressed to Yongsheng Yang; ysyang@jlu.edu.cn and Xuewen Zhang; zhangxw@jlu.edu.cn
}

Received 24 August 2017; Revised 2 January 2018; Accepted 21 January 2018; Published 26 March 2018

Academic Editor: Qing Lin

Copyright (C) 2018 Yingjun Xie et al. This is an open access article distributed under the Creative Commons Attribution License, which permits unrestricted use, distribution, and reproduction in any medium, provided the original work is properly cited.

\begin{abstract}
Primary liver cancer is a common kind of digestive cancers with high malignancy, causing 745,500 deaths each year. Hepatocellular carcinoma is the major pathological type of primary liver cancer. Traditional treatment methods for patients with hepatocellular carcinoma have shown poor efficacy in killing residual cancer cells for a long time. In recent years, tumor immunotherapy has emerged as a promising method owing to its safety and efficacy with respect to delaying the progression of advanced tumors and protecting postoperative patients against tumor relapse and metastasis. Immune tolerance and suppression in tumor microenvironments are the theoretical basis of immunotherapy. Adoptive cell therapy functions by stimulating and cultivating autologous lymphocytes ex vivo and then reinfusing them into the patient to kill cancer cells. Cancer vaccination is performed using antigenic substances to activate tumor-specific immune responses. Immune checkpoint inhibitors can reactivate tumorspecific T cells and develop an antitumor effect by suppressing checkpoint-mediated signaling. Oncolytic viruses may selectively replicate in tumor cells and cause lysis without harming normal tissues. Here, we briefly introduce the mechanism of immunosuppression in hepatocellular carcinoma and summarize the rationale of the four major immunotherapeutic approaches with their current advances.
\end{abstract}

\section{Introduction}

Primary liver cancer is the sixth most common type of cancer and the second most common cause of cancer-related deaths worldwide, with an extremely high malignancy such that the number of deaths $(745,500)$ is similar to that of new cases $(782,500)$ every year [1]. Hepatocellular carcinoma (HCC) is a predominant type of primary liver cancer. Traditional therapeutic approaches for HCC include radical or palliative liver resection, radioactive seed implantation, transarterial chemoembolization (TACE), radiofrequency ablation (RFA), and liver transplantation. Although these approaches effectively address local lesions, they fail to completely eliminate residual cancer cells, which lead to tumor recurrence and metastasis. In recent years, tumor immunotherapy has emerged as a promising method for inhibiting tumor progression, relapse, and metastasis [2]. The rationale of this method is to activate tumor-specific immune responses and disrupt immune tolerance by enhancing cellular or humoral immunity. To date, some immunotherapeutic drugs for treating hematological malignancies, melanomas, and lung cancers have been proven to be efficacious in phase III trials and have been approved by FDA. Furthermore, recently, studies on immunotherapeutic approaches for HCC are rapidly increasing. In this study, we briefly reviewed the mechanism underlying immunosuppression and summarized major immunotherapeutic approaches for HCC (Table 1).

\section{Mechanism Underlying Immunosuppression in HCC}

$\mathrm{T}$ cells are activated through a double signaling pathway that requires the interaction of T cell receptors (TCR) with major histocompatibility complex (MHC)/peptide complexes on antigen-presenting cells (APCs) and expression of costimulatory molecules (CMs) on T cells and APCs. Downregulation 
TABLE 1: Major immunotherapeutic approaches for HCC.

\begin{tabular}{lcr}
\hline Approaches & Subsets & Targets and applications \\
\hline \multirow{2}{*}{ ACT } & CIK cells & CIK with valproate, DC-CIK with TACE \\
& TILs & NK cells \\
& CAR T cells (generations 1-4) & NK with K562-mb15-41BBL, sorafenib, and NKG2D \\
& Cell vaccines & Targeting GPC3, targeting GPC3 and ASGR1 \\
HCC vaccines & Antigen peptide vaccines & AFP, GPC3, SSX-2, NY-ESO-1, hTERT, HCA587, and MAGE-A \\
& DC vaccines & TCL-loaded DCs with nifuroxazide \\
\hline Immune checkpoint inhibitors & CTLA-4 inhibitors & Tremelimumab, Tremelimumab with RFA \\
& PD-1 inhibitors & Nivolumab, Pembrolizumab, and Pidilizumab \\
\hline Oncolytic viruses & PD-L1 inhibitors & PD-L1 inhibitor with DNMT1 inhibitor \\
\hline
\end{tabular}

of MHC class I molecules on tumor cells induces impairment of tumor antigen processing and presentation [3, 4]. Furthermore, reduced expression of CMs, such as B7-1 and B7-2, in HCC [4] leads to T cell anergy.

Immune checkpoints normally protect humans from uncontrolled autologous immunity by preventing excessive activation of T cells. However, tumor cells can overexpress immune checkpoint molecules that bind their receptors on $\mathrm{T}$ cells and inhibit $\mathrm{T}$ cell activation. The upregulation of immune checkpoint pathways in most patients with HCC impairs the effector function of cellular immune responses [5-7].

Immunosuppression in HCC can also be achieved via impairment of $\mathrm{CD}^{+} \mathrm{T}$ cells [8]. MHC class II genes are among the most frequently expressed genes in HCC tumors, and overexpression of MHC class II molecules leads to CD $4^{+}$ $\mathrm{T}$ cell anergy in the absence of suitable CMs [9]. Also, immunosuppressive cells, including T regulatory cells (Tregs) [10], myeloid-derived suppressor cells (MDSCs) [5], and regulatory dendritic cells (DCs) [11], are important immunosuppressive factors in cancer patients, and an increase in the number of immunosuppressive cells, such as Tregs, may contribute to disease progression and poor prognosis. A Th1/Th2-like cytokine shift in the liver microenvironment of HCC patients with venous metastases has been previously reported [12]. Moreover, the upregulation of anti-inflammatory/immunosuppressive Th2-like cytokines and downregulation of proinflammatory/immunogenic Th1-like cytokines in adjacent noncancerous hepatic tissues indicate that disordered immune responses in tumor microenvironments [13-15] are key predictors of HCC metastasis.

\section{Adoptive Cell Therapy}

Adoptive cell therapy (ACT) is an immunotherapeutic approach that kills cancer cells using patients' own lymphocytes. It functions by stimulating or loading autologous lymphocytes with cytokines or tumor antigens, cultivating them ex vivo and then reinfusing them into the patient [16-18]. Adoptive immunotherapy for HCC includes cytokineinduced killer (CIK) cells, tumor-infiltrating lymphocytes (TILs), natural killer (NK) cells, and chimeric antigen receptor (CAR) T cells. The feasibility and safety of ACT in patients with HCC have been evaluated in many experiments, thus laying a foundation for its clinical application.

3.1. CIK Cells. CIK cells are a heterogeneous MHCindependent cell population mainly comprising $\mathrm{CD}^{+-}$ $\mathrm{CD}_{56}{ }^{+}, \mathrm{CD}^{+} \mathrm{CD}^{-} 6^{-}$, and $\mathrm{CD}^{-} \mathrm{CD}^{+} 6^{+}$cells [19-21]. CIK cells are derived from peripheral mononuclear cells and stimulated by IL-1, IL-12, interferon- (IFN-) $\gamma$, and anti-CD3 antibodies ex vivo [22]. In a phase III study of adjuvant CIK therapy after radical resection for HCC, patients were randomized to receive four cycles of CIK therapy or no treatment. The median time to recurrence (TTR) was 13.6 months in the CIK group and 7.8 months in the control group $(p=0.01)$, indicating the safety and efficacy with respect to prolonging TTR of CIK therapy in patients with HCC. However, there were no statistically significant differences between the groups in disease-free survival (DFS) and overall survival (OS) [23]. A combination therapy with CIK cells and valproate in mice demonstrated a synergistic effect in controlling tumor growth [24], warranting further assessment of this combination therapy through clinical trials. In addition, a meta-analysis of 693 patients with HCC demonstrated that a combination of dendritic cell- (DC-) CIK cells and TACE improves 1- and 2-year OS, overall response rate (ORR), disease control rate (DCR), and the quality of life [25].

3.2. TILs. TILs are derived from tumor tissues and are cultured and induced using IL-2 and anti-CD3 antibodies ex vivo [26-28]. Thus, reinfusion of autologous TILs, which possess tumor-specific immunity, may target multiple tumor antigens. Low toxicity of autologous TILs was verified in a phase I study involving patients with HCC, suggesting a novel treatment option [29]. However, this study included only 15 patients and lacked control groups, thus failing to prove the efficacy of TILs. To date, TILs have not been well characterized, mainly due to difficulties in purifying and expanding them.

3.3. NK Cells. NK cells belong to the innate immune system and can directly kill tumor cells and infected cells without preliminary sensitization or MHC restriction. However, they 


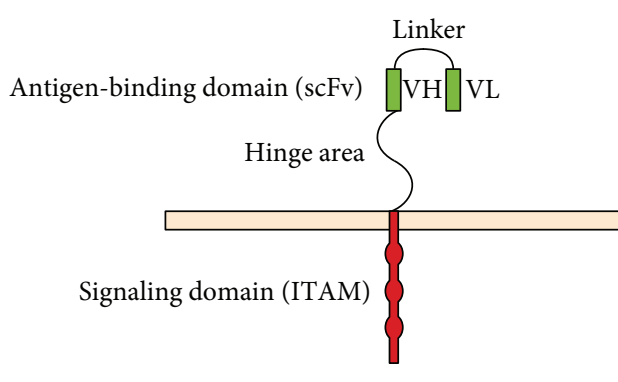

Basic structure of CAR

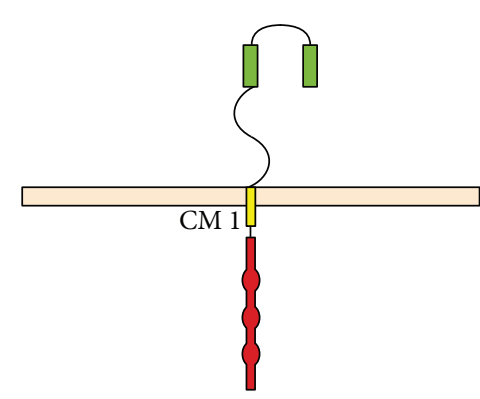

Second-generation CAR

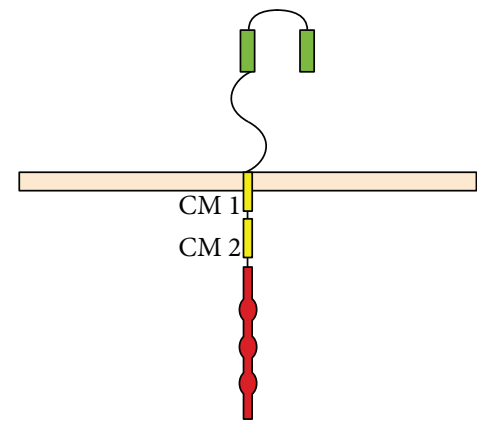

Third-generation CAR

FIgURe 1: Structure of CAR.

lack the ability to target tumor cells and can injure normal liver tissues. In a previous series of experiments, the cytotoxicity of NK cells against HCC cells was enhanced [30] by first generating a new hepatoma cell line, K562-mb15-41BBL, which achieved a more efficient stimulation of NK cells in vitro. Second, HCC cells exposed to $5 \mu \mathrm{mol} / \mathrm{L}$ sorafenib for $48 \mathrm{~h}$ showed high sensitivity to NK cells. Finally, NKG2D, an engineered NK-cell-activating receptor, was tested in vitro and in mice. All of the outcomes were positive in increasing the cytotoxicity of NK cells, providing the possibility of further clinical trials for HCC.

3.4. CAR T Cells. CAR $\mathrm{T}$ cells are genetically modified $\mathrm{T}$ lymphocytes that specifically target tumor-associated antigens (TAAs) and kill cancer cells in a MHC-independent manner $[31,32]$. CARs consist of three major components-the extracellular antigen-binding domain, the intracellular signaling domain, and the hinge area [33,34] (Figure 1). The antigen-binding domain is a single chain fragment variable $(\mathrm{scFv})$ region that comprises a heavy $(\mathrm{VH})$ and a light (VL) chain derived from monoclonal antibodies (mAbs), which are connected by a linker fragment. The signaling domain involves immunoreceptor tyrosine-based activation motifs (ITAMs), such as CD3 $\zeta$ and FceRI $\gamma$. The above two domains are connected by the hinge area which imparts high flexibility for the movement of the antigen-binding domain. The first-generation CARs lacked the structure of CMs and led to poor replication, survival, and cytotoxicity of T cells. In contrast, the second- and third-generation CARs (Figure 1), with the addition of CMs, led to high proliferative capacity, long-term persistence, and potent cytotoxicity of $\mathrm{T}$ cells [35]. In the second-generation CARs, a CM (CM1), such as $\mathrm{CD} 28$, was engineered into the signaling region [36], whereas in the third generation, additional CMs (CM2), such as CD27, CD137/4-1BB, and CD134/OX40, were included [37]. In some tumors with a tremendous phenotypic heterogeneity, CAR $\mathrm{T}$ cells could target the tumor antigen and cause antigen-positive cell death, while antigen-negative cancer cells may induce tumor relapse. Recently, CAR T cells with a transgenic "payload," also called the "fourthgeneration" CAR T cells, were designed [38]. The fourthgeneration CAR $T$ cells work by releasing inducible cytokines such as IL-12 which will augment $\mathrm{T}$ cell activation and further activate innate immune system to kill antigennegative cancer cells. Recently, CAR $\mathrm{T}$ cell therapy has received much attention as an immunotherapy for tumors, and a good efficacy has been reported in some clinical trials of leukemia and lymphoma. CAR T cell therapy is also being investigated for solid tumors, such as HCC. Glypican3 (GPC3) is a TAA that is specifically overexpressed in $70 \%-$ $81 \%$ of HCC tumors and has been correlated with poor prognosis [39]. Moreover, the ability of GPC3-targeted CAR T cells to eliminate GPC3-positive HCC cells was confirmed both in vivo and in vitro, and the survival of mice with HCC xenografts was evidently prolonged with CAR T cell therapy in vivo [40]. In another experiment, $T$ cells with two complementary CARs against GPC 3 and asialoglycoprotein receptor 1 (ASGR1) decreased the risk of on-target, offtumor toxicities and demonstrated potent antitumor immune responses targeting GPC3+ ASGR1+ HCCs both in vivo and in vitro [41]. However, to date, the related studies conducted have been predominantly basic, and more clinical trials are required to prove the efficacy of CAR $\mathrm{T}$ cells against HCC. Complications of CAR T cell therapy include on-target, off-tumor toxicities [42], tumor lysis syndrome (TLS) [43], and cytokine release syndrome (CRS) [44]. Traditional solutions include nonspecific immunosuppression, complete elimination of $\mathrm{T}$ cells, and introduction of inducible suicide genes into CAR T cells. However, the best method to prevent these hazards could be the application of ideal tumor-specific antigens (TSAs) expressed only in tumor cells and not in normal cells. As for the difficulty of seeking for more appropriate TSAs, some techniques, such as the utilization of inhibitory CAR (iCAR) [45] and combinatorial antigen recognition by CAR and chimeric costimulatory receptor (CCR) [46], were employed to prevent on-target, off-tumor toxicities. In addition, CARs could be used to modify other lymphocytes, such as NK cells [47] and $\gamma \delta$ T cells [48], which may highlight the use of HCC immunotherapy in the future.

\section{HCC Vaccines}

Cancer vaccination is performed using antigenic substances to activate tumor-specific immune responses that can reduce tumor load and prevent tumor relapse. HCC vaccines include cancer cells, antigen peptides, DCs, and DNA-based 
vaccines, and some of these effectively inhibit tumor recurrence and metastasis.

4.1. HCC Cell Vaccines. Autologous or allogenic HCC cells or lysates that are physically or chemically disposed to eliminate pathogenicity could be used as immunogens for tumor-specific immune responses. In a phase I trial, bishRNA/granulocyte macrophage colony-stimulating factor(GM-CSF-) augmented autologous tumor cells were tested in eight patients with advanced HCC. Three of these patients presented evident immune responses to the reinfused tumor cells, and long-term follow-up demonstrated a survival of $319,729,784,931+$, and 1043+ days after treatment [49]. However, the efficacy of HCC cell vaccines remains uncertain due to their weak immunogenicity.

4.2. Antigen Peptide Vaccines. Peptide-based TAAs, such as alpha-fetoprotein (AFP), GPC3, SSX-2, NY-ESO-1, human telomerase reverse transcriptase (hTERT), HCA587, and melanoma antigen gene-A (MAGE-A), are excellent vaccine targets for the treatment of HCC [50]. AFP, which normally originates from embryonic liver cells, can be overexpressed on HCC cell surfaces. However, immune responses to AFP are limited due to acquired immune tolerance during the development of the immune system. To break up this immune tolerance, a research group investigated the use of a recombinant rat AFP to induce cross-reactions between xenografts and endogenous molecules in animals and observed modest cellular and humoral immune responses [51]. In a phase II trial of GPC3-derived peptide vaccine for HCC, 25 patients received 10 vaccinations over one year after surgery. Recurrence in patients who underwent both surgery and vaccination was specifically lower than that in 21 patients who underwent surgery only (24\% versus $48 \%$ and $52.4 \%$ versus $61.9 \%$ at 1 and 2 years, $p=0.047$ and 0.387 , resp.), indicating the efficacy of the GPC3-derived vaccine [52].

4.3. DC Vaccines. DCs, the most powerful APCs, are responsible for absorption, processing, and presentation of tumor antigens. They maintain high expression levels of MHCs and CMs, such as B7-1 and B7-2. They also elicit antitumor effects by the way of inducing primary $\mathrm{T}$ cells, releasing IFN- $\gamma$ that suppresses tumoral angiogenesis and producing immune memory [53]. During vaccine preparation, DCs are initially activated by cytokines, such as rhGM-CSF and rhIL-4, then mature in the presence of tumor necrosis factor(TNF-) $\alpha$ and are finally sensitized by autologous tumor cells or antigens [50]. Some gene-transfected DCs persistently express endogenous tumor antigens or cytokines that enhance their own functions. In a recent study, mice with HCC were treated with a combination of tumor cell lysate(TCL-) loaded DCs and nifuroxazide, which is an inhibitor of signal transducer and activator of transcription 3 (STAT3). This combination increased the survival rate, limited tumor growth, and elevated antitumor immune response [54]. A phase I/IIa study using tumor antigen-pulsed DCs for HCC patients after primary treatment demonstrated that DC vaccination is an effective adjuvant treatment for such patients
[55]. In addition, the safety and tolerance of DC vaccines have been confirmed in patients with HCC [56].

\section{Immune Checkpoint Inhibitors}

As mentioned above, the negative regulatory target-immune checkpoints are often overexpressed in tumors to escape the host immune surveillance. Immune checkpoint inhibitors can reactivate tumor-specific T cells and develop an antitumor effect by suppressing checkpoint-mediated signaling [57]. Common immune checkpoint proteins include cytotoxic $\mathrm{T}$ lymphocyte-associated antigen-4 (CTLA-4), programmed cell death protein-1 (PD-1), programmed cell death ligand 1 (PD-L1), VISTA, TIM-3, LAG-3, and OX40 [58, 59]. CTLA-4 and PD-1 inhibitors have been well characterized and have been approved by FDA for treating melanomas, with some progress in their application in treating HCCs.

5.1. CTLA-4 Inhibitors. CTLA-4 is predominantly expressed in activated $\mathrm{T}$ cells and NK cells [60]. It binds ligands B7-1 and B7-2 with much higher affinity than CD28 [61]. Moreover, CTLA-4 inhibitors prevent the binding of CTLA-4 to B7-1 and B7-2, thereby promoting the activation of T cells. In 2011, FDA approved a fully human anti-CTLA-4 mAbIpilimumab for the treatment of metastatic melanoma. In a phase II study of an anti-CTLA-4 mAb-Tremelimumab in patients with advanced $\mathrm{HCC}$ and hepatitis $\mathrm{C}$, partial response rate $(17.6 \%)$, disease control rate $(76.4 \%)$, and time to progression (6.48 months) improved. Moreover, viral loads of HCC were significantly decreased, and no patients experienced immune-related adverse events (irAEs) or evident hepatotoxicity. These studies demonstrated that Tremelimumab treatment is a safe antitumor and antiviral method for hepatitis C-induced HCC [62]. In a noncomparative clinical trial involving patients with advanced HCC, a combination therapy with Tremelimumab and RFA increased the number of intratumoral $\mathrm{CD}^{+} \mathrm{T}$ cells and reduced HCV viral loads [63].

5.2. PD-1 Inhibitors. PD-1 is expressed in T cells, B cells, NK cells, mononuclear cells, and DCs [64]. PD-1 inhibitors block the receptor binding of PD-L1 and PD-L2, resulting in the activation of immune cells [65]. Some PD-1 inhibitors, such as Nivolumab, Pembrolizumab, and Pidilizumab, have been investigated for cancer treatment. A phase I/II study demonstrated the safety and antitumor effect of Nivolumab in patients with advanced HCC. In this study, of the 41 qualified patients who were intravenously administered $0.1-10 \mathrm{mg} / \mathrm{kg}$ Nivolumab, 29 (71\%, 17\% grade 3/4) endured drug-related AEs, two (5\%) showed complete responses (CRs), and seven (18\%) showed partial responses (PRs). Moreover, response durations for CR, PR, and stable disease (SD) were 14-17+ months, $<1-8+$ months, and 1.5-17+ months, respectively, and the OS rate at 6 months was $72 \%$. These data indicated that Nivolumab activates sustained tumor-specific immune responses with manageable AEs [66]. A recent open-label, noncomparative, phase I/II dose escalation and expansion trial of Nivolumab involving 262 patients with advanced 
HCC confirmed the safety and potential of this PD-1 inhibitor in treating HCCs [67].

5.3. PD-L1 Inhibitors. Cancer cells can evade immune surveillance by overexpressing PD-L1 and activating PDL1/PD-1 signaling [68]. High PD-L1 expression has been observed in HCC tissues [69]. However, no clinical trials involving the use of PD-L1 inhibitors for treating HCC have been conducted. A recent experiment showed that contemporary inhibition of PD-L1 and DNA methyltransferase 1 (DNMT1) significantly suppressed the growth of sorafenibresistant HCC cells in vitro, further suggesting a novel effective treatment option for sorafenib-resistant HCC [70].

\section{Oncolytic Virotherapy}

Oncolytic viruses are wild-type or engineered viruses that selectively replicate in tumor cells and cause lysis without harming normal tissues [71, 72]. The mechanism underlying the antitumor activity of oncolytic viruses involves direct killing of cancer cells by expanding in them and causing cell lysis. Most viruses can expand in cancer cells to a rather great extent due to the impairment of the tumor's defense mechanisms against viral infection [73]. In addition, tumor antigens and viruses in cell lysates activate immune responses against adjacent cancer cells [74-77]. The targeting mechanisms of oncolytic viruses are as follows. First, wild-type viruses that specifically infect tumors like reoviruses, varicella viruses, and Sindbis viruses [78] could be chosen. Second, viral genes that are crucial for replication in normal cells but have no functions in cancer cells are deleted by engineering [76]. Third, viral transcription is limited in cancer cells by applying tumor-specific promoters, such as the promoter of human telomerase reverse transcriptase, before crucial viral genes [79]. Finally, after modification by TAA-specific receptors, viruses effectively target tumor cells. For example, an oncolytic vaccinia virus engineered with antiangiogenic genes can specifically inhibit tumor angiogenesis [80]. The efficacy of an evolutionary cancer-favoring engineered vaccinia virus (CVV) was investigated in an animal model of metastatic HCC. In this study, animals were randomized into sorafenib, CVV, and sorafenib with CVV groups. Metastatic regions were fewer in the CVV-treated groups than in the sorafenib-treated group. The result suggested that CVV can be a promising virus targeting metastatic HCC [81]. JX-594, an engineered vaccinia virus with a mutation in the TK gene, which controls cancer cell-specific replication, and an insertion in the human GM-CSF gene, which increases antitumor immune responses [82], is stable and safe in humans and extremely toxic to cancer cells. A phase II randomized open-label study of JX-594 in patients with advanced HCC confirmed the safety and efficacy of the oncolytic virotherapy. This treatment was well tolerated at both high and low doses, with an intrahepatic response rate of $62 \%$ and one CR. In addition, the OS rate was higher in the high-dose group than in the low-dose group (median, 14.1 months versus 6.7 months; hazard ratio, $0.39 ; p=0.020$ ) [83]. To date, various oncolytic viruses, such as GLV-1h68 [84] and G47delta [85], have been studied for the treatment of HCC.
Researchers should attach more importance to the dangers of viral infection and the insertional mutations that may activate oncogenes or damage tumor suppressor genes.

\section{Brief Summary}

The four major immunotherapeutic approaches for HCC have their own preponderances and defects.

CAR $T$ cell therapy has been a star of immunotherapeutic researches in recent years. With its accurate targeting toward HCC and MHC independence, CAR T cells could directively kill HCC cells, like precision-guided missiles. The efficacy of CAR T cells has also been elevated after several generations. However, this favored method is not almighty. The lack of HCC-associated TSAs makes it difficult to construct more efficacious CARs. Meanwhile, more strategies should be designed to overcome the on-target, off-tumor effect. Other methods of adoptive cell therapy, like CIK cells, TILs, and NK cells, are being out of sight due to the nonspecificity and difficulty of extraction.

Immune checkpoint inhibitor is another hot topic. It breaks up tumor immune tolerance and causes reactivation of innate immune system, which may redirect and eliminate HCC cells as a result. It is a relatively simple process preparing for immune checkpoint inhibitors. Meanwhile, many clinical researches indicate the safety of this method. So, we may focus on how to improve its efficacy and test more practical combinatorial therapeutic methods in the future.

Tumor vaccines, because of tumor immune tolerance and lack of TSAs, did not show great value in HCC treatment, while DC vaccines may be a promising method in this realm, due to their potent capacity of antigen presenting. Researches of oncolytic viruses are quite few. Safety of viruses is the most important, while efficacy is the second. So the very much difficulty is to balance safety and toxicity of oncolytic viruses.

\section{Future Expectations}

As a new therapeutic approach for malignancies beyond traditional operations, chemotherapy and radiotherapy, immunotherapy has shown its efficacy in delaying the progression of advanced tumors and protecting postoperative patients against cancer relapse and metastasis. Although no drugs have been officially approved, numerous studies on immunotherapy for HCC are being conducted and some have already obtained important results. Future studies are required to identify more specific immune targets, such as TAAs/TSAs, novel immune checkpoints, and oncolytic viruses. These will enhance the intensity of tumor-specific immune responses and avoid unnecessary on-target, offtumor toxicities. Meanwhile, the Aes should be valued, especially in clinical trials. The safety of a new treatment is as important as its efficacy. Furthermore, individualized treatment plans for patients with HCC will enhance the efficacy of immunotherapy and likely become a future trend. Taken together, the promising therapeutic approach certainly will bring the treatment for HCC to a brand new period. 


\section{Conflicts of Interest}

The authors confirm no conflicts of interest regarding the publication of this article.

\section{Acknowledgments}

This work was supported by grants from the Finance Department of Jilin Province (SCZSYZ01502, SCZSYZ01521, and SCZSYZ01522).

\section{References}

[1] L. A. Torre, F. Bray, R. L. Siegel, J. Ferlay, J. Lortet-Tieulent, and A. Jemal, "Global cancer statistics, 2012," CA: A Cancer Journal for Clinicians, vol. 65, no. 2, pp. 87-108, 2015.

[2] L. X. Qin, "Inflammatory immune responses in tumor microenvironment and metastasis of hepatocellular carcinoma," Cancer Microenvironment, vol. 5, no. 3, pp. 203-209, 2012.

[3] N. Tsuchiya, Y. Sawada, I. Endo, Y. Uemura, and T. Nakatsura, "Potentiality of immunotherapy against hepatocellular carcinoma," World Journal of Gastroenterology, vol. 21, no. 36, pp. 10314-10326, 2015.

[4] K. Fujiwara, T. Higashi, K. Nouso et al., "Decreased expression of B7 costimulatory molecules and major histocompatibility complex class-I in human hepatocellular carcinoma," Journal of Gastroenterology and Hepatology, vol. 19, no. 10, pp. 1121-1127, 2004.

[5] F. Arihara, E. Mizukoshi, M. Kitahara et al., "Increase in CD $14^{+}$HLA-DR ${ }^{-/ \text {low }}$ myeloid-derived suppressor cells in hepatocellular carcinoma patients and its impact on prognosis," Cancer Immunology, Immunotherapy, vol. 62, no. 8, pp. 1421-1430, 2013.

[6] Z. Chen, S. Shen, B. Peng, and J. Tao, "Intratumoural GM-CSF microspheres and CTLA-4 blockade enhance the antitumour immunity induced by thermal ablation in a subcutaneous murine hepatoma model," International Journal of Hyperthermia, vol. 25, no. 5, pp. 374-382, 2009.

[7] B. Hoechst, T. Voigtlaender, L. Ormandy et al., "Myeloid derived suppressor cells inhibit natural killer cells in patients with hepatocellular carcinoma via the NKp30 receptor," Hepatology, vol. 50, no. 3, pp. 799-807, 2009.

[8] A. Alisa, A. Ives, A. A. Pathan et al., "Analysis of CD4 $4^{+}$T-cell responses to a novel $\alpha$-fetoprotein-derived epitope in hepatocellular carcinoma patients," Clinical Cancer Research, vol. 11, no. 18, pp. 6686-6694, 2005.

[9] I. N. Crispe, M. Giannandrea, I. Klein, B. John, B. Sampson, and S. Wuensch, "Cellular and molecular mechanisms of liver tolerance," Immunological Reviews, vol. 213, no. 1, pp. 101118, 2006.

[10] K. J. Chen, S. Z. Lin, L. Zhou et al., "Selective recruitment of regulatory $\mathrm{T}$ cell through CCR6-CCL20 in hepatocellular carcinoma fosters tumor progression and predicts poor prognosis," PLoS One, vol. 6, no. 9, article e24671, 2011.

[11] Y. Han, Z. Chen, Y. Yang et al., "Human CD14 ${ }^{+}$CTLA- $4^{+}$ regulatory dendritic cells suppress $\mathrm{T}$-cell response by cytotoxic T-lymphocyte antigen-4-dependent IL-10 and indoleamine2,3-dioxygenase production in hepatocellular carcinoma," Hepatology, vol. 59, no. 2, pp. 567-579, 2014.

[12] A. Budhu, M. Forgues, Q. H. Ye et al., "Prediction of venous metastases, recurrence, and prognosis in hepatocellular carcinoma based on a unique immune response signature of the liver microenvironment," Cancer Cell, vol. 10, no. 2, pp. 99-111, 2006.

[13] J. Couzin-Frankel, "Breakthrough of the year 2013. Cancer immunotherapy," Science, vol. 342, no. 6165, pp. 1432-1433, 2013.

[14] F. M. Buonaguro and L. Buonaguro, "Cancer vaccines for hepatocellular carcinoma: future directions," Immunotherapy, vol. 8, no. 4, pp. 391-393, 2016.

[15] L. Buonaguro, A. Petrizzo, M. Tagliamonte, M. L. Tornesello, and F. M. Buonaguro, "Challenges in cancer vaccine development for hepatocellular carcinoma," Journal of Hepatology, vol. 59, no. 4, pp. 897-903, 2013.

[16] Z. Jixia, Z. Chengyan, and W. Pingli, "Advances in application of adoptive T-cell therapy for cancer patients," Zhejiang Da Xue Xue Bao Yi Xue Ban, vol. 46, no. 2, pp. 211-217, 2017.

[17] O. Yeku, X. Li, and R. J. Brentjens, “Adoptive T-cell therapy for solid tumors," American Society of Clinical Oncology Educational Book, vol. 37, pp. 193-204, 2017.

[18] E. N. Baruch, A. L. Berg, M. J. Besser, J. Schachter, and G. Markel, "Adoptive T cell therapy: an overview of obstacles and opportunities," Cancer, vol. 123, no. S11, pp. 2154-2162, 2017.

[19] V. Longo, A. Gnoni, A. Casadei Gardini et al., "Immunotherapeutic approaches for hepatocellular carcinoma," Oncotarget, vol. 8, no. 20, pp. 33897-33910, 2017.

[20] X. Gao, Y. Mi, N. Guo et al., "Cytokine-induced killer cells as pharmacological tools for cancer immunotherapy," Frontiers in Immunology, vol. 8, p. 774, 2017.

[21] J. J. Mata-Molanes, M. Sureda Gonzalez, B. Valenzuela Jimenez, E. M. Martinez Navarro, and A. Brugarolas Masllorens, "Cancer immunotherapy with cytokine-induced killer cells," Targeted Oncology, vol. 12, no. 3, pp. 289-299, 2017.

[22] F. S. Wang, M. X. Liu, B. Zhang et al., "Antitumor activities of human autologous cytokine-induced killer (CIK) cells against hepatocellular carcinoma cells in vitro and in vivo," World Journal of Gastroenterology, vol. 8, no. 3, pp. 464-468, 2002.

[23] L. Xu, J. Wang, Y. Kim et al., "A randomized controlled trial on patients with or without adjuvant autologous cytokineinduced killer cells after curative resection for hepatocellular carcinoma," OncoImmunology, vol. 5, no. 3, article e1083671, 2016.

[24] D. H. Lee, J. Y. Nam, Y. Chang et al., "Synergistic effect of cytokine-induced killer cell with valproate inhibits growth of hepatocellular carcinoma cell in a mouse model," Cancer Biology \& Therapy, vol. 18, no. 1, pp. 67-75, 2017.

[25] Y. Su, Y. Yang, Y. Ma et al., "The efficacy and safety of dendritic cells co-cultured with cytokine-induced killer cell therapy in combination with TACE-predominant minimallyinvasive treatment for hepatocellular carcinoma: a meta-analysis," Clinical Laboratory, vol. 62, no. 4, pp. 599-608, 2016.

[26] U. Toh, T. Fujii, N. Seki, F. Niiya, K. Shirouzu, and H. Yamana, "Characterization of IL-2-activated TILs and their use in intrapericardial immunotherapy in malignant pericardial effusion," Cancer Immunology, Immunotherapy, vol. 55, no. 10, pp. 1219-1227, 2006.

[27] L. Yuan, S. He, C. Guan, and Q. Pang, “The preparation and study on hepatic targeting tendency of galactosyl-anti-CD3McAb in mice," Hua Xi Yi Ke Da Xue Xue Bao, vol. 32, no. 3, pp. 424-426, 2001.

[28] T. Kikuchi, M. Watanabe, and T. Ohno, "Cytological characteristics of human glioma-infiltrating lymphocytes stimulated 
with recombinant interleukin 2 and an anti-CD3 antibody," Japanese Journal of Cancer Research, vol. 82, no. 3, pp. 339-345, 1991.

[29] S. S. Jiang, Y. Tang, Y. J. Zhang et al., “A phase I clinical trial utilizing autologous tumor-infiltrating lymphocytes in patients with primary hepatocellular carcinoma," Oncotarget, vol. 6, no. 38, pp. 41339-41349, 2015.

[30] T. Kamiya, Y. H. Chang, and D. Campana, "Expanded and activated natural killer cells for immunotherapy of hepatocellular carcinoma," Cancer Immunology Research, vol. 4, no. 7, pp. 574-581, 2016.

[31] M. Chmielewski, A. A. Hombach, and H. Abken, "Antigenspecific T-cell activation independently of the MHC: chimeric antigen receptor-redirected T cells," Frontiers in Immunology, vol. 4, p. 371, 2013.

[32] T. Li, H. T. Wang, and Z. G. Liu, "CAR technology and its application in treatment of multiple myeloma-review," Zhongguo Shi Yan Xue Ye Xue Za Zhi, vol. 24, no. 1, pp. 279-284, 2016.

[33] C. A. Ramos and G. Dotti, "Chimeric antigen receptor (CAR)engineered lymphocytes for cancer therapy," Expert Opinion on Biological Therapy, vol. 11, no. 7, pp. 855-873, 2011.

[34] S. J. Priceman, S. J. Forman, and C. E. Brown, "Smart CARs engineered for cancer immunotherapy," Current Opinion in Oncology, vol. 27, no. 6, pp. 466-474, 2015.

[35] G. Dotti, B. Savoldo, and M. Brenner, "Fifteen years of gene therapy based on chimeric antigen receptors: "are we nearly there yet?"," Human Gene Therapy, vol. 20, no. 11, pp. 1229-1239, 2009.

[36] S. J. C. van der Stegen, M. Hamieh, and M. Sadelain, “The pharmacology of second-generation chimeric antigen receptors," Nature Reviews Drug Discovery, vol. 14, no. 7, pp. 499-509, 2015.

[37] X. Y. Tang, Y. Sun, A. Zhang et al., “Third-generation CD28/4$1 \mathrm{BB}$ chimeric antigen receptor $\mathrm{T}$ cells for chemotherapy relapsed or refractory acute lymphoblastic leukaemia: a nonrandomised, open-label phase I trial protocol," BMJ Open, vol. 6, no. 12, article e013904, 2016.

[38] M. Chmielewski and H. Abken, "TRUCKs: the fourth generation of CARs," Expert Opinion on Biological Therapy, vol. 15, no. 8, pp. 1145-1154, 2015.

[39] H. Shirakawa, H. Suzuki, M. Shimomura et al., "Glypican-3 expression is correlated with poor prognosis in hepatocellular carcinoma," Cancer Science, vol. 100, no. 8, pp. 1403-1407, 2009.

[40] H. Gao, K. Li, H. Tu et al., "Development of T cells redirected to glypican-3 for the treatment of hepatocellular carcinoma," Clinical Cancer Research, vol. 20, no. 24, pp. 6418-6428, 2014.

[41] C. Chen, K. Li, H. Jiang et al., "Development of T cells carrying two complementary chimeric antigen receptors against glypican-3 and asialoglycoprotein receptor 1 for the treatment of hepatocellular carcinoma," Cancer Immunology, Immunotherapy, vol. 66, no. 4, pp. 475-489, 2017.

[42] G. Gross and Z. Eshhar, "Therapeutic potential of T cell chimeric antigen receptors (CARs) in cancer treatment: counteracting off-tumor toxicities for safe CAR T cell therapy," Annual Review of Pharmacology and Toxicology, vol. 56, no. 1, pp. 59-83, 2016.

[43] D. T. Teachey, S. F. Lacey, P. A. Shaw et al., "Identification of predictive biomarkers for cytokine release syndrome after chimeric antigen receptor T-cell therapy for acute lymphoblastic leukemia," Cancer Discovery, vol. 6, no. 6, pp. 664-679, 2016.

[44] X. J. Xu and Y. M. Tang, "Cytokine release syndrome in cancer immunotherapy with chimeric antigen receptor engineered $\mathrm{T}$ cells," Cancer Letters, vol. 343, no. 2, pp. 172-178, 2014.

[45] V. D. Fedorov, M. Themeli, and M. Sadelain, "PD-1- and CTLA-4-based inhibitory chimeric antigen receptors (iCARs) divert off-target immunotherapy responses," Science Translational Medicine, vol. 5, no. 215, article 215ra172, 2013.

[46] C. C. Kloss, M. Condomines, M. Cartellieri, M. Bachmann, and M. Sadelain, "Combinatorial antigen recognition with balanced signaling promotes selective tumor eradication by engineered T cells," Nature Biotechnology, vol. 31, no. 1, pp. 71-75, 2013.

[47] H. J. Pegram, J. T. Jackson, M. J. Smyth, M. H. Kershaw, and P. K. Darcy, "Adoptive transfer of gene-modified primary NK cells can specifically inhibit tumor progression in vivo," Journal of Immunology, vol. 181, no. 5, pp. 3449-3455, 2008.

[48] M. Rischer, S. Pscherer, S. Duwe, J. Vormoor, H. Jurgens, and C. Rossig, "Human $\gamma \delta \mathrm{T}$ cells as mediators of chimaericreceptor redirected anti-tumour immunity," British Journal of Haematology, vol. 126, no. 4, pp. 583-592, 2004.

[49] J. Nemunaitis, M. Barve, D. Orr et al., "Summary of bi-shRNAfurin/GM-CSF augmented autologous tumor cell immunotherapy $\left(\mathrm{FANG}^{\mathrm{TM}}\right)$ in advanced cancer of the liver," Oncology, vol. 87, no. 1, pp. 21-29, 2014.

[50] T. Y. Sun, W. Yan, C. M. Yang et al., "Clinical research on dendritic cell vaccines to prevent postoperative recurrence and metastasis of liver cancer," Genetics and Molecular Research, vol. 14, no. 4, pp. 16222-16232, 2015.

[51] W. Zhang, J. Liu, Y. Wu et al., "Immunotherapy of hepatocellular carcinoma with a vaccine based on xenogeneic homologous $\alpha$ fetoprotein in mice," Biochemical and Biophysical Research Communications, vol. 376, no. 1, pp. 10-14, 2008.

[52] Y. Sawada, T. Yoshikawa, K. Ofuji et al., "Phase II study of the GPC3-derived peptide vaccine as an adjuvant therapy for hepatocellular carcinoma patients," OncoImmunology, vol. 5, no. 5, article e1129483, 2016.

[53] K. Takakura, M. Kajihara, Z. Ito, T. Ohkusa, J. Gong, and S. Koido, "Dendritic-tumor fusion cells in cancer immunotherapy," Discovery Medicine, vol. 19, no. 104, pp. 169-174, 2015.

[54] T. Zhao, H. Jia, Q. Cheng et al., "Nifuroxazide prompts antitumor immune response of TCL-loaded DC in mice with orthotopically-implanted hepatocarcinoma," Oncology Reports, vol. 37, no. 6, pp. 3405-3414, 2017.

[55] J. H. Lee, Y. Lee, M. Lee et al., "A phase I/IIa study of adjuvant immunotherapy with tumour antigen-pulsed dendritic cells in patients with hepatocellular carcinoma," British Journal of Cancer, vol. 113, no. 12, pp. 1666-1676, 2015.

[56] M. El Ansary, S. Mogawer, S. A. Elhamid et al., "Immunotherapy by autologous dendritic cell vaccine in patients with advanced HCC," Journal of Cancer Research and Clinical Oncology, vol. 139, no. 1, pp. 39-48, 2013.

[57] J. B. Haanen and C. Robert, "Immune checkpoint inhibitors," Progress in Tumor Research, vol. 42, pp. 55-66, 2015.

[58] T. Hato, L. Goyal, T. F. Greten, D. G. Duda, and A. X. Zhu, "Immune checkpoint blockade in hepatocellular carcinoma: current progress and future directions," Hepatology, vol. 60, no. 5, pp. 1776-1782, 2014. 
[59] X. Meng, Z. Huang, F. Teng, L. Xing, and J. Yu, "Predictive biomarkers in PD-1/PD-L1 checkpoint blockade immunotherapy," Cancer Treatment Reviews, vol. 41, no. 10, pp. 868-876, 2015.

[60] M. D. Vesely, M. H. Kershaw, R. D. Schreiber, and M. J. Smyth, "Natural innate and adaptive immunity to cancer," Annual Review of Immunology, vol. 29, no. 1, pp. 235-271, 2011.

[61] A. V. Collins, D. W. Brodie, R. J. C. Gilbert et al., "The interaction properties of costimulatory molecules revisited," Immunity, vol. 17, no. 2, pp. 201-210, 2002.

[62] B. Sangro, C. Gomez-Martin, M. de la Mata et al., "A clinical trial of CTLA-4 blockade with tremelimumab in patients with hepatocellular carcinoma and chronic hepatitis C," Journal of Hepatology, vol. 59, no. 1, pp. 81-88, 2013.

[63] A. G. Duffy, S. V. Ulahannan, O. Makorova-Rusher et al., "Tremelimumab in combination with ablation in patients with advanced hepatocellular carcinoma," Journal of Hepatology, vol. 66, no. 3, pp. 545-551, 2017.

[64] L. Shi, S. Chen, L. Yang, and Y. Li, "The role of PD-1 and PD-L1 in T-cell immune suppression in patients with hematological malignancies," Journal of Hematology \& Oncology, vol. 6, no. 1, p. 74, 2013.

[65] S. Dai, R. Jia, X. Zhang, Q. Fang, and L. Huang, "The PD-1/PDLs pathway and autoimmune diseases," Cellular Immunology, vol. 290, no. 1, pp. 72-79, 2014.

[66] A. B. El-Khoueiry, I. Melero, T. S. Crocenzi et al., "Phase I/II safety and antitumor activity of nivolumab in patients with advanced hepatocellular carcinoma (HCC): CA209-040," Journal of Clinical Oncology, vol. 33, no. 18, article LBA101, 2015 Supplement, 2015.

[67] A. B. El-Khoueiry, B. Sangro, T. Yau et al., "Nivolumab in patients with advanced hepatocellular carcinoma (CheckMate 040): an open-label, non-comparative, phase 1/2 dose escalation and expansion trial," Lancet, vol. 389, no. 10088, pp. 2492-2502, 2017.

[68] Y. Iwai, M. Ishida, Y. Tanaka, T. Okazaki, T. Honjo, and N. Minato, "Involvement of PD-L1 on tumor cells in the escape from host immune system and tumor immunotherapy by PD-L1 blockade," Proceedings of the National Academy of Sciences of the United States of America, vol. 99, no. 19, pp. 12293-12297, 2002.

[69] F. Zhong, X. Cheng, S. Sun, and J. Zhou, "Transcriptional activation of PD-L1 by Sox 2 contributes to the proliferation of hepatocellular carcinoma cells," Oncology Reports, vol. 37, no. 5, pp. 3061-3067, 2017.

[70] J. Liu, Y. Liu, L. Meng, K. Liu, and B. Ji, “Targeting the PD-L1/ DNMT1 axis in acquired resistance to sorafenib in human hepatocellular carcinoma," Oncology Reports, vol. 38, no. 2, pp. 899-907, 2017.

[71] A. M. Hammill, J. Conner, and T. P. Cripe, "Oncolytic virotherapy reaches adolescence," Pediatric Blood \& Cancer, vol. 55, no. 7, pp. 1253-1263, 2010.

[72] M. G. Bourke, S. Salwa, K. J. Harrington et al., "The emerging role of viruses in the treatment of solid tumours," Cancer Treatment Reviews, vol. 37, no. 8, pp. 618-632, 2011.

[73] L. C. Platanias, "Mechanisms of type-I- and type-II-interferonmediated signalling," Nature Reviews Immunology, vol. 5, no. 5, pp. 375-386, 2005.

[74] H. L. Kaufman, F. J. Kohlhapp, and A. Zloza, "Oncolytic viruses: a new class of immunotherapy drugs," Nature Reviews Drug Discovery, vol. 14, no. 9, pp. 642-662, 2015.
[75] V. Schirrmacher, "Oncolytic Newcastle disease virus as a prospective anti-cancer therapy. A biologic agent with potential to break therapy resistance," Expert Opinion on Biological Therapy, vol. 15, no. 12, pp. 1757-1771, 2015.

[76] E. A. Chiocca and S. D. Rabkin, "Oncolytic viruses and their application to cancer immunotherapy," Cancer Immunology Research, vol. 2, no. 4, pp. 295-300, 2014.

[77] S. T. Workenhe and K. L. Mossman, "Oncolytic virotherapy and immunogenic cancer cell death: sharpening the sword for improved cancer treatment strategies," Molecular Therapy, vol. 22, no. 2, pp. 251-256, 2014.

[78] M. M. Stanford, J. C. Bell, and M. J. V. Vähä-Koskela, "Novel oncolytic viruses: riding high on the next wave?," Cytokine \& Growth Factor Reviews, vol. 21, no. 2-3, pp. 177-183, 2010.

[79] W. Zhang, K. Ge, Q. Zhao et al., "A novel oHSV-1 targeting telomerase reverse transcriptase-positive cancer cells via tumor-specific promoters regulating the expression of ICP4," Oncotarget, vol. 6, no. 24, pp. 20345-20355, 2015.

[80] C. J. Breitbach, R. Arulanandam, N. de Silva et al., "Oncolytic vaccinia virus disrupts tumor-associated vasculature in humans," Cancer Research, vol. 73, no. 4, pp. 1265-1275, 2013.

[81] S. Y. Yoo, S. N. Jeong, D. H. Kang, and J. Heo, "Evolutionary cancer-favoring engineered vaccinia virus for metastatic hepatocellular carcinoma," Oncotarget, vol. 8, no. 42, pp. 7148971499, 2017.

[82] K. A. Parato, C. J. Breitbach, F. le Boeuf et al., "The oncolytic poxvirus JX-594 selectively replicates in and destroys cancer cells driven by genetic pathways commonly activated in cancers," Molecular Therapy, vol. 20, no. 4, pp. 749-758, 2012.

[83] C. J. Breitbach, A. Moon, J. Burke, T. H. Hwang, and D. H. Kirn, "A phase 2, open-label, randomized study of Pexa-Vec (JX-594) administered by intratumoral injection in patients with unresectable primary hepatocellular carcinoma," Methods in Molecular Biology, vol. 1317, pp. 343-357, 2015.

[84] J. W. Ady, J. Heffner, K. Mojica et al., "Oncolytic immunotherapy using recombinant vaccinia virus GLV-1h68 kills sorafenib-resistant hepatocellular carcinoma efficiently," Surgery, vol. 156, no. 2, pp. 263-269, 2014.

[85] J. Wang, L. Xu, W. Zeng et al., "Treatment of human hepatocellular carcinoma by the oncolytic herpes simplex virus G47delta," Cancer Cell International, vol. 14, no. 1, p. 83, 2014. 


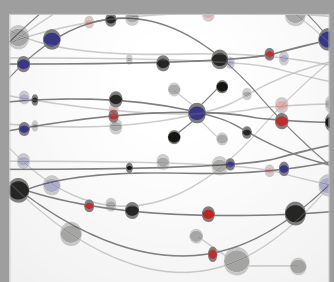

The Scientific World Journal
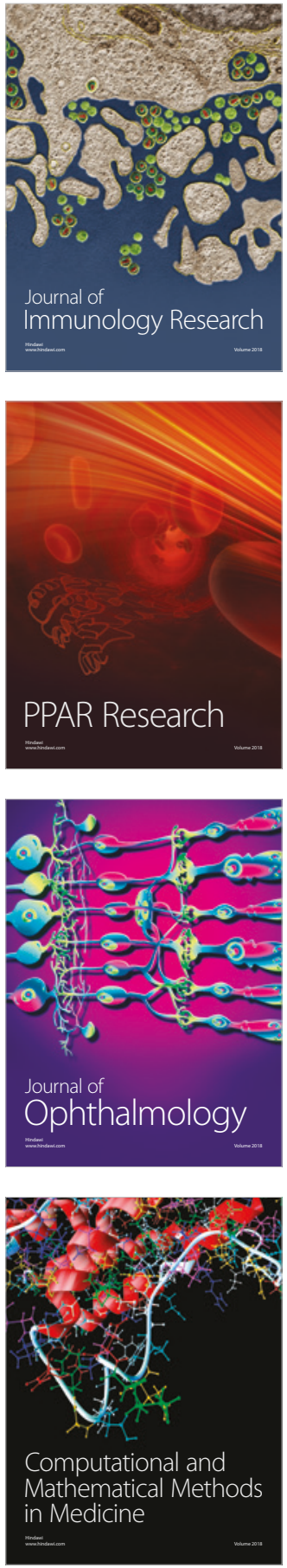

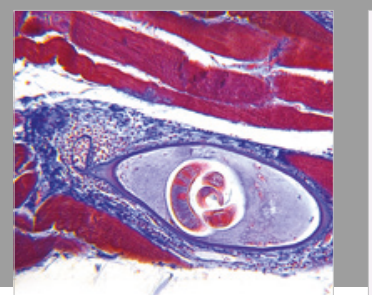

Gastroenterology Research and Practice

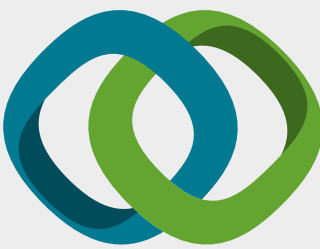

\section{Hindawi}

Submit your manuscripts at

www.hindawi.com
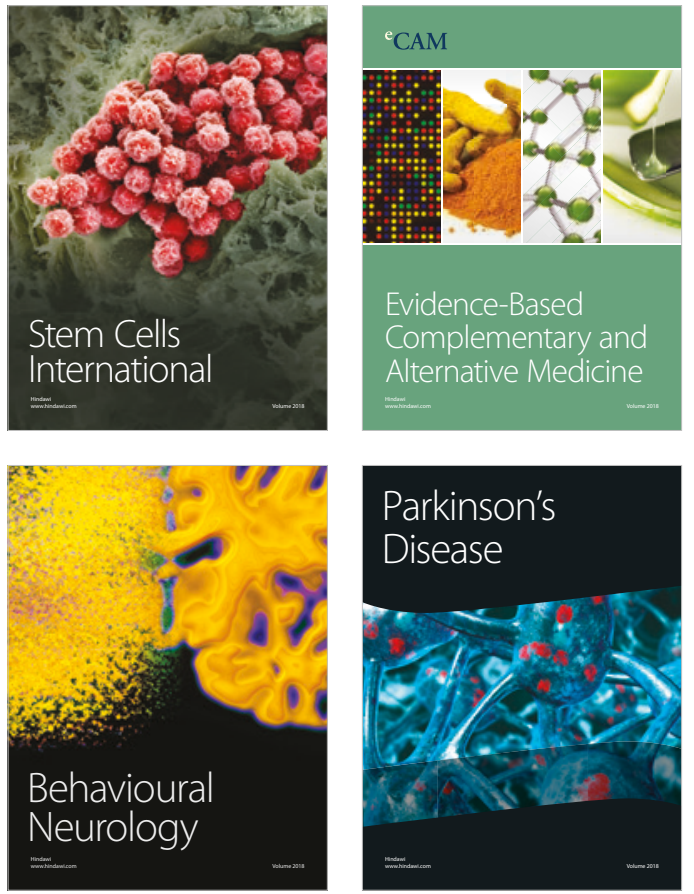

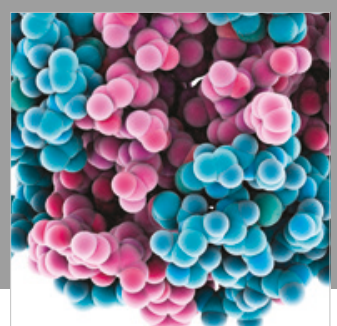

ournal of

Diabetes Research

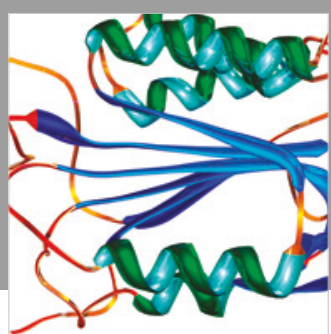

Disease Markers
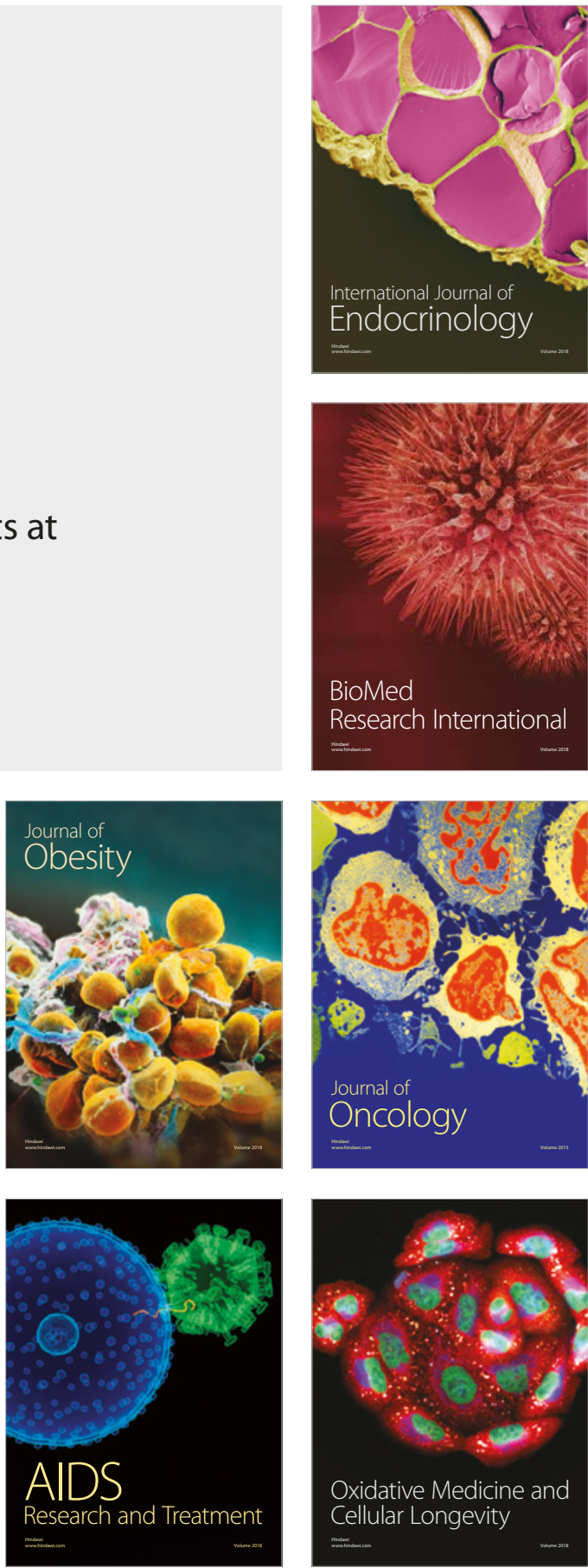